\title{
Assessment of Inflammatory Biomarkers, Total IgE levels, SNOT-22 scores in Allergic Rhinitis patients
}

\author{
Dr. Demet Yazıcı \\ ENT Department, Adana City Training and Research Hospital, Adana \\ ORCID NO: 0000-0002-5726-1987
}

\begin{abstract}
Objective: The aim of this study is to assess neutrophil-lymphocyte ratio (NLR), mean platelet volume (MPV), platelet-lymphocyte ratio (PLR) and eosinophil-lymphocyte ratio (ELR) of allergic rhinitis patients and compare our findings with healthy controls. We also analyzed the effect of severity of allergic rhinitis on hematological parameters and total IgE levels of the patients.
\end{abstract}

Methods: A total of forty-six patients diagnosed having allergic rhinitis according to Allergic Rhinitis and its Impact on Asthma (ARIA) criteria and forty-six healthy, age and sex-matched subjects were included in this study. The clinical records of the patients were retrospectively analyzed and age, gender, skin prick test results, complete blood counts, total IgE levels and Sino-Nasal Outcome Test (SNOT) 22 scores were recorded for assessment of neutrophil-lymphocyte ratio (NLR), mean platelet volume (MPV), platelet-lymphocyte ratio (PLR) and eosinophil-lymphocyte ratio (ELR) of the patients.
Results: Eosinophil count, eosinophil percentage and ELR value of the study group were significantly higher than the control group ( $p=0.001, p=0.001, p=0.001$, respectively). The cut-off value for ELR was $\geq 0.105$ (sensitivity $=0.61$, specificity $=0.76, A \cup C=0.703$ ). Total IgE levels of patients with positive skin prick test were significantly higher than patients with negative skin prick test $(p=0.019)$. The cutoff value for total $\mathrm{IgE}$ was $\geq 63 \mathrm{IU} / \mathrm{mL}$ (sensitivity $=0.59$, specificity $=0.74, A \cup C=0.706$ ).

Conclusion: ELR is a valuable marker for predicting allergic rhinitis. However, it does not correlate with the severity of the disease. Total IgE levels increase in patients with positive skin prick tests. Also, total IgE level does not correlate with the severity of allergic rhinitis.

Keywords: Allergic rhinitis, biomarkers, eosinophils, lymphocytes.

\section{Introduction}

Allergic rhinitis is a type 1 hypersensitivity reaction with an increasing prevalence around the world. ARIA (Allergic rhinitis and its impact on asthma) guideline defines allergic rhinitis as "rhinorrhoea, nasal itching, nasal obstruction and sneezing which are reversible spontaneously or with treatment". ${ }^{[1]}$ The aeroallergens causing rhinitis can be identi- fied by skin prick tests, nasal provocation tests (NPT) and specific IgE levels in blood and in nasal secretions. ${ }^{[2]}$ Nasal cytology also can be helpful in diagnosing allergic rhinitis.

Among all these diagnostic methods, skin prick test (SPT) is the most widely used cost-effective test for detection of type 1 hypersensitivity. Helmtraud Ebruster demon-
Correspondence: Dr. Demet Yazıc1

ENT Department, Adana City Training and Research Hospital, Adana email: demetyazici@yahoo.com
Online available at: www.entupdates.org

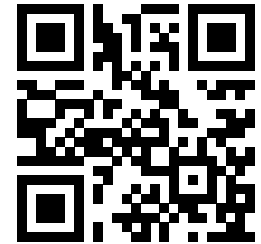


strated the feasibility of this test in 1959 , and since then, SPT is not only used in allergic rhinitis, but also in the diagnosis of allergic diseases such as atopic dermatitis, allergic rhinoconjunctivitis, asthma and food and drug allergy ${ }^{[3,4]}$ Other diagnostic tests such as NPT and specific $\mathrm{IgE}$ levels in blood and nasal secretions are all costly tests with lower sensitivity. ${ }^{[2]}$

Neutrophil-lymphocyte ratio (NLR), mean platelet volume (MPV), eosinophil-lymphocyte ratio (ELR) and platelet-lymphocyte ratio (PLR) are systemic chronic inflammatory biomarkers used as prognosticators and predictors in oncology, systemic autoimmune rheumatic diseases, cardiovascular and allergic diseases. They are costeffective and easy to calculate, thus attracting researchers in various areas.

In this study, we compared neutrophil-lymphocyte ratio (NLR), mean platelet volume (MPV), eosinophillymphocyte ratio (ELR), platelet-lymphocyte ratio (PLR), eosinophil count and eosinophil percentage of allergic rhinitis patients with healthy subjects and tried to determine a cutoff value for predicting allergic rhinitis. We divided allergic rhinitis patients into two groups as skin prick test (SPT) positive and negative patients and assessed the difference between them by means of total $\mathrm{IgE}$ values, SNOT-22 scores, eosinophil count, eosinophil percentage and ELR values.

\section{Methods}

The clinical records of patients diagnosed as persistent allergic rhinitis having skin prick tests were analyzed retrospectively. The Allergic Rhinitis and its Impact on Asthma (ARIA-2008) guidelines were used for the diagnose of allergic rhinitis. ${ }^{[1]}$ Patients with nasal polyposis, systemic diseases, asthma, parasitic infections, other atopic diseases and having history of recent use of antiallergic drugs were excluded from the study. The sensitivity for aeroallergens was tested by skin prick test (SPT) and the severity of the disease was measured by Turkish translation of the 22 -item Sino-Nasal Outcome Test (SNOT-22 test). ${ }^{[5]}$ SPT test was applied on right or left forearm of patients with test applicators. SPT was performed by using 8 most common allergen extracts of our region with standard activity and concentration. (Allergopharma, prick test kit) The SPT also included positive (histamine) and negative (physiological saline) controls. The allergen extracts used in SPT were as follows: house dust mite (Dermatophagoides pteronyssinus, Dermatophagoides farina), animal epithelia (cat, dog), mold
(Cladosporium herbarum), grass pollen mix (orchard grass, velvet grass, Timothy grass, Kentucky blue grass, rye grass, meadow fescue), grass and cereal pollen mix (grass pollen mix with barley, oat, rye, wheat) and tree pollen mix (hazel, elm, poplar, willow tree, alder). The wheal diameter was evaluated after 20 minutes and a wheal diameter $3 \mathrm{~mm}$ greater than the control was regarded as a positive result. The control group consisted of healthy individuals without allergic rhinitis. Approval of ethical committee was obtained from our institution.

Complete blood count (CBC) with total $\mathrm{IgE}$ level was obtained from all allergic rhinitis patients before SPT. Neutrophil count, platelet count, lymphocyte count, eosinophil count, eosinophil percentage and mean platelet volume (MPV) of each patient was noted and neutrophil-lymphocyte ratio (NLR) was calculated by dividing neutrophil count to lymphocyte count $\left(\mathrm{NLR}=\right.$ no. of neutrophils $\left(10^{3}\right.$ $\mathrm{mcL}) /$ no. of lymphocytes $\left(10^{3} \mathrm{mcL}\right)$ ), platelet-lymphocyte ratio (PLR) was calculated by dividing platelet count to lymphocyte count $\left(\mathrm{PLR}=\right.$ no of platelets $\left(10^{3} \mathrm{mcL}\right) /$ no. of lymphocytes $\left(10^{3} \mathrm{mcL}\right)$ ) and eosinophil-lymphocyte ratio (ELR) was calculated by dividing eosinophil count to lymphocyte count $\left(\mathrm{ELR}=\right.$ no. of eosinophils $\left(10^{3} \mathrm{mcL}\right) / \mathrm{no}$. of lymphocytes $\left.\left(10^{3} \mathrm{mcL}\right)\right)$.

SPSS for windows 20.0 (IBM) was used for data analysis. Chi-square and Mann-Whitney $\mathrm{U}$ test were used for statistical analysis. Multivariate logistic regression analysis was used to identify independent risk factors and ROC analysis was done to calculate the cut off value for variables with sensitivity and specificity. A p value lesser than 0.05 was considered statistically significant. For correlation of the variables, Spearman correlation analysis was used to analyze the relationship between SNOT scores, total IgE and ELR.

\section{Results}

Forty-six patients full-filled the criteria of this study. The control group was composed of 46 healthy, age and sex -matched subjects without allergic rhinitis. The demographic characteristics of the study and the control group along with mean eosinophil count, eosinophil percentage, NLR, PLR, MPV and ELR value is listed in Table 1. As seen in Table 1, there was a statistically significant difference between eosinophil count, eosinophil percentage and ELR values of the study and control group ( $\mathrm{p}=0.001$, $\mathrm{p}=0.001, \mathrm{p}=0.001$ respectively). 


\begin{tabular}{|c|c|c|c|}
\hline & Study Group & Control group & $P$ value \\
\hline \multirow[b]{2}{*}{ Gender } & $23(50 \%)$ & $23(50 \%)$ & \multirow{3}{*}{$p=1.0$} \\
\hline & & & \\
\hline Female & $23(50 \%)$ & $23(50 \%)$ & \\
\hline Age (median) years & $31.5(\min 16-\max 65)$ & $27(\min 18-\max 56)$ & $p=0.407$ \\
\hline Eosinophil count (mean \pm SD) $103:<\mathrm{L}$ & $0.37 \pm 0.4$ & $0.17 \pm 0.1$ & $P=0.001$ \\
\hline Eosinophil \% (mean \pm SD) & $4.59 \pm 3.7$ & $2.41 \pm 1.5$ & $P=0.001$ \\
\hline NLR $($ mean $\pm S D)$ & $2.04 \pm 0.7$ & $1.93 \pm 0.7$ & $P=0.295$ \\
\hline $\mathrm{PLR}($ mean $\pm \mathrm{SD})$ & $120.8 \pm 36.9$ & $119.9 \pm 36.8$ & $P=0.978$ \\
\hline MPV $($ mean \pm SD) & $8.83 \pm 1.1$ & $8.68 \pm 0.9$ & $P=0.472$ \\
\hline ELR $($ mean $\pm S D)$ & $0.16 \pm 0.14$ & $0.08 \pm 0.06$ & $P=0.001$ \\
\hline
\end{tabular}

We also performed univariate and multivariate logistic regression analysis of NLR, PLR and ELR for predicting allergic rhinitis. According to univariate and multivariate analysis, ELR was the only independent predictor for allergic rhinitis $(\mathrm{p}=0.002)$. The risk for allergic rhinitis increases 3.118 times in univariate and 3.168 times multivariate analysis as the ELR increases $(\mathrm{OR}=3.118, \mathrm{OR}=3.168 \mathrm{re}-$ spectively). The cut-off value for ELR was $\geq 0.105$ (sensitivity $=0.61$, specificity $=0.76, \mathrm{AUC}=0.703$ ) .

Table 2: Univariate and multivariate analysis of the effect of NLR, PLR and ELR on allergic rhinitis (Cl: confidence interval; OR: odds ratio; NLR: neutrophil/lymphocyte ratio; PLR: platelet/lymphocyte ratio; ELR eosinophil/lymphocyte ratio)

\begin{tabular}{ccccc}
\hline & Univariate & \multicolumn{3}{c}{ Multivariate } \\
\hline & OR $(\% 95 \mathrm{Cl})$ & $\mathbf{p}$ & OR $(\% 95 \mathrm{Cl})$ & $\mathbf{p}$ \\
\hline NLR & $1.18(0.778-1.788)$ & 0.436 & $1.178(0.749-1.852)$ & 0.479 \\
PLR & $1.022(0.678-1.542)$ & 0.916 & $0.883(0.554-1.406)$ & 0.600 \\
ELR & $3.118(1.523-6.383)$ & 0.002 & $3.168(1.521-6.596)$ & 0.002 \\
\hline
\end{tabular}

The demographic characteristics, total IgE levels, SNOT-22 test outcomes, mean eosinophil count, eosinophil percentage and ELR values of the SPT positive and SPT negative patients are demonstrated at Table 3 . As seen in Table 3, there was a statistically significant difference between total IgE levels of the SPT positive and negative group.

We performed ROC analysis for determining SPT positivity and the cut-off value for total $\mathrm{IgE}$ was $\geq 63 \mathrm{IU} / \mathrm{mL}$ (sensitivity $=0.59$, specificity $=0.74, \mathrm{AUC}=0.706$ ).
When we analyzed the correlation between SNOT scores and total IgE levels and SNOT scores and ELR values of SPT positive and negative patients, there was no statistically significant association between them $(\mathrm{p}=0.244$, $\mathrm{p}=0.630$ respectively).

Of the 27 patients with positive SPT test, only 5 of them had sensitization to one allergen, 22 of them were polysensitized. The most common allergen positivity was against Dermatophagoides pteronyssinus (48.1\%), Dermatophagoides farinea (44.4\%), grass and cereal pollen $(40.7 \%)$ and $\operatorname{dogs}(33.3 \%)$.

\section{Discussion}

Allergic rhinitis is an IgE-mediated type 1 hypersensitivity reaction of the nasal mucosa. This hypersensitivity reaction may be against only one allergen (monosensitized) or two or more allergens (polysensitized). Skin prick tests (SPT), specific IgE levels in blood may be used to identify these allergens or SPT may be negative in patients with local allergic rhinitis so nasal provocation test (NPT) or nasal specific IgE might be useful. ${ }^{[6]}$ NLR, PLR, mean platelet volume (MPV) and ELR are well-known, easily-calculated, low-cost inflammatory biomarkers. NLR, PLR, MPV and ELR are all well-studied biomarkers of oncologic, autoimmune and cardiac diseases. ELR is also linked with allergic airway diseases in literature.

In rhinology, NLR, PLR and ELR are analyzed for their significance in predicting recurrence of nasal polyposis after endoscopic sinus surgery. In the study of Boztepe et al, NLR and PLR was studied and NLR was revealed as the only risk factor for recurrence of nasal polyposis. ${ }^{[7]}$ In the 


\begin{tabular}{|c|c|c|c|}
\hline & SPT positive & SPT negative & $P$ value \\
\hline \multirow{2}{*}{ Gender } & $13 / 27$ & $10 / 19$ & \multirow{2}{*}{$\mathrm{p}=0.765$} \\
\hline & $14 / 27$ & $9 / 19$ & \\
\hline Age (median) years & $31(\min 17-\max 65)$ & $32(\min 16-\max 46)$ & $p=0.867$ \\
\hline Total IgE (IU/mL) & $68(\min 13-\max 2148)$ & $37(\min 12-\max 620)$ & $P=0.019$ \\
\hline SNOT-22 & $45.2 \pm 16$ & $46.5 \pm 20$ & $P=0.816$ \\
\hline Eosinophil count (mean \pm SD) $103<<$ & $0.37 \pm 0.37$ & $0.37 \pm 0.38$ & $P=0.955$ \\
\hline Eosinophil \% (mean \pm SD) & $4.93 \pm 4.1$ & $4.09 \pm 3.1$ & $P=0.532$ \\
\hline $\operatorname{ELR}($ mean $\pm S D)$ & $0.17 \pm 0.16$ & $0.14 \pm 0.12$ & $P=0.704$ \\
\hline
\end{tabular}

study of Brescia et al, NLR, ELR and basophil-lymphocyte ratio (BLR) was evaluated and NLR, ELR and BLR were significantly higher in patients with recurrence. ${ }^{[8]}$ In the study of Yenigun, NLR and ELR were again found higher in patients with recurrent nasal polyposis after endoscopic sinus surgery. ${ }^{[9]}$

NLR, ELR and eosinophil count also were assessed in several studies about allergic rhinitis. In the study of Dogru et al, NLR was significantly higher in children diagnosed as allergic rhinitis compared with the control group and when the allergic rhinitis patients were classified according to their symptoms as mild and moderate/severe group, NLR was even higher in the moderate/severe group. ${ }^{[10]}$ In the study of Yenigun et al, ELR and eosinophil count was higher in SPT positive patients compared with SPT negative patients. ${ }^{[11]}$ In our study, eosinophil count, eosinophil percentage and ELR values of the allergic rhinitis patients were higher than the control group. However, when we divided patients into two groups as SPT positive and SPT negative, although SPT positive group had higher eosinophil percentage and ELR values, the difference between two groups was not statistically significant. Our study group consisted only of patients with persistent allergic rhinitis, however in the study of Yenigün et al, patients were classified as being symptomatic and asymptomatic and the duration of allergic rhinitis was not specified. ${ }^{[1]}$

In the study of Pinar et al, total Ig E levels of allergic rhinitis patients with positive prick tests were compared with control group composed of healthy subjects and there was statistically significant difference between them. ${ }^{[12]}$ The study of Chen et al assessed the correlation between total nasal symptom score and total IgE, allergen specific $\mathrm{IgE}$, eosinophil cationic protein (ECP), blood and nasal eosinophil count in children with perennial allergic rhinitis and found out all these markers correlated with severity of rhinitis. ${ }^{[13]}$ In the study of Hyun et al ${ }^{[14]}$, total $\operatorname{IgE}$ value and serum eosinophil count of allergic rhinitis and nonallergic rhinitis patients were compared and total $\operatorname{IgE}$ value and serum eosinophil count of allergic rhinitis patients were found out to be higher than nonallergic rhinitis patients. Also, total IgE values and serum eosinophil count positively correlated with the number of allergens positive on skin prick test. $^{[14]}$ In the study of Demirjian et a ${ }^{[15]}$, total IgE values of SPT positive and negative allergic rhinitis patients with or without asthma were compared and mean total $\mathrm{IgE}$ value of SPT positive rhinitis patients with asthma was $175 \mathrm{IU} / \mathrm{ml}$, SPT positive rhinitis patients without asthma was $87 \mathrm{IU} / \mathrm{ml}$, SPT negative rhinitis patients with asthma $40 \mathrm{IU} / \mathrm{ml}$ and SPT negative rhinitis patients without asthma was $23 \mathrm{IU} / \mathrm{ml}$. They also concluded a threshold level of $140 \mathrm{IU} / \mathrm{ml}$ for total $\mathrm{IgE}$ in SPT positive patients.

${ }^{[15]}$ In our study, mean total IgE value for SPT positive patients was $68 \mathrm{IU} / \mathrm{ml}$ and for SPT negative patients $37 \mathrm{IU} /$ $\mathrm{ml}$ and cut-off value for total $\mathrm{IgE}$ was $\geq 63 \mathrm{IU} / \mathrm{mL}$. All the patients included in our study were diagnosed as persistent allergic rhinitis according to ARIA. None of our patients had asthma. We also couldn't find any correlation between SNOT-22 scores and the other hematological parameters. However, total $\mathrm{IgE}$ values of SPT positive group were higher than SPT negative group. $(\mathrm{p}=0.019)$

In this study, we found out that elevated ELR was the only inflammatory biomarker in allergic rhinitis and an 
ELR value greater than 0.105 is a predictor for this disease. Also, total IgE value above $63 \mathrm{IU} / \mathrm{mL}$ is suggestive of positivity in skin prick test. Our study is one of the few studies in literature comparing many inflammatory biomarkers between allergic and nonallergic patients, also giving a cut-

\section{References}

1. Bousquet J,Khaltaev N, Cruz AA, et al. World Health Organization, GA(2)LEN, AllerGen. Allergic Rhinitis and its Impact on Asthma (ARIA) 2008 update (in collaboration with the World Health Organization, GA(2)LEN and AllerGen). Allergy 2008;63:8-160.

2. Incorvaia C, Fuiano N, Martignago I, Gritti BL, Ridolo E. Local allergic rhinitis: evolution of concepts. Clin Transl Allergy 2017;7:38.

3. Ebruster H. The prick test, a recent cutaneous test for the diagnosis of allergic disorders. [Article in German] Wien Klin Wochenschr 1959;71:551-4.

4. Heinzerling L, Mari A, Bergmann KC, et al. The skin prick test European standards. Clin Trans Allergy 2013;3:3.

5. Hancı D, Altun H, Şahin E, Altıntoprak N, Cingi C. Turkish translation, cross-cultural adaptation and validation of the SinoNasal Outcome Test (SNOT)-22. ENT Updates 2015;5:51-7.

6. Campo P, Rondón C, Gould HJ, Barrionuevo E, Gevaert P, Blanca M. Local IgE in non-allergic rhinitis. Clin Exp Allergy 2015;45:872-81.

7. Boztepe OF, Gün T, Demir M, et al. A novel predictive marker for the recurrence of nasal polyposis following endoscopic sinus surgery. Eur Arch Otorhinolaryngol 2016;273:1439-44.

8. Brescia G, Pedruzzi B, Barion U, et al. Are neutrophil-, eosinophil-, and basophil-to-lymphocyte ratios useful markers for pinpointing patients at higher risk of recurrent sinonasal polyps? Am J Otolaryngol 2016;37:339-45. off value for total $\mathrm{IgE}$ for determining allergic rhinitis and skin prick test positivity. As our study group is relatively small, more studies with larger patient groups is needed for better evaluation.
9. Yenigün A. Assessment of patients with nasal polyposis by the neutrophiltolymphocyte ratio and eosinophil-to-lymphocyte ratio. Kulak Burun Bogaz Ihtis Derg 2015;25:193-9.

10. Dogru M, Evcimik MF, Cirik AA. Is neutrophil-lymphocyte ratio associated with the severity of allergic rhinitis in children? Eur Arch Otorhinolaryngol 2016;273:3175-8.

11. Yenigun A, Sezen S, Calim OF, Ozturan O. Evaluation of the Eosinophiltolymphocyte Ratio in Pediatric Patients with Allergic Rhinitis. Am J Rhinol Allergy 2016;30:21-5.

12. Pınar E, Bolat F, Öncel S, Köseoğlu M, Yüksel H, Calli C. The relationship between skin test results and serum eosinophilic cationic protein, nasal eosinophilia, and total IgE values in patients with allergic rhinitis. [Article in Turkish] Kulak Burun Bogaz Ihtis Derg 2005;14:101-5.

13. Chen ST, Sun HL, Lu KH, Lue KH, Chou MC. Correlation of immunoglobulin E, eosinophil cationic protein, and eosinophil count with the severity of childhood perennial allergic rhinitis. J Microbiol Immunol Infect 2006;39:212-8.

14. Hyun JM, Young HH, Hoon SY, Kyung SK. The correlation of serum eosinophil cationic protein level with eosinophil count, and total IgE level in Korean adult allergic rhinitis patients. Asian Pac J Allergy Immunol 2016;34:290-4.

15. Demirjian M, Rumbyrt JS, Gowda VC, Klaustermeyer WB. Serum IgE and eosinophil count in allergic rhinitis-Analysis using a modified Bayes' theorem. Allergol Immunopathol (Madr) 2012;40:281-7. 